\title{
EVALUACIÓN DE TÉCNICAS PARA LA REDUCCIÓN DE LA CONCENTRACIÓN DE SÓLIDOS SEDIMENTABLES EN LOS VERTIMIENTOS PRODUCIDOS EN LA MINERÍA DE CARBÓN EN EL MUNICIPIO DE GUACHETÁ, CUNDINAMARCA
}

\section{Evaluation of techniques to reduce the concentration of sedimentable solids in the wastewater produced in the coal mining in the municipality of Guachetá, Cundinamarca}

\author{
Sandra Liliana Chaparro Alba \\ Universidad Manuela Beltrán, Programa de Ingeniería Ambiental, Colombia. \\ Email: alba.sandraliliana@gmail.com
}

(Recibido el 10 de febrero de 2021 - Aceptado el 18 de junio de 2021)

\section{Resumen}

La filtración de aguas residuales superficiales y subterráneas es muy común cuando se avanzan labores de explotación minera, ya que se deben cruzar acuíferos subterráneos, bloques antiguos explotados y, dentro del proceso de extracción, se genera un asentamiento de los estratos que producen algún rango de subsidencia y fracturamiento, dando lugar a la filtración de estas aguas desde la superficie del terreno al interior de las minas. Las aguas residuales deben ser evacuadas nuevamente hacia la superficie, para lo cual es necesario conducirlas a lo largo de las labores mineras (utilizando mecanismos como cunetas, mangueras y tuberías), proceso que tiende a contaminar el ambiente de polución minera, inclusive, con el contacto directo de las rocas de las excavaciones. Una vez conducidas a la superficie, por medio de electrobombas de gran capacidad y caudal, y dependiendo de la profundidad de las labores, estas aguas residuales deben ser manejadas en sistemas de tratamiento con el fin de realizar una limpieza, en este caso de las concentraciones de sólidos sedimentables y poder ser vertidas a las fuentes cercanas, para ser reutilizadas en procesos diferentes al consumo humano. El presente estudio evaluó de manera técnica, económica y ambiental, tres alternativas de tratamiento de aguas, donde se obtuvo resultados de la alternativa 1 Coagulación-Floculación, un porcentaje de eficiencia del 99,12\%, un costo total de instalación de $\$ 17 ’ 363.978$ pesos y una afectación ambiental baja, simulado con el software SuperDecisions ${ }^{\circledR}$ que la Alternativa 1 Coagulación-Floculación tiene el ranking número 1, seguido de la alternativa Carbón Activado con ranking número 2 y, por último, el mantenimiento del sistema con ranking 3.

Palabras Clave: aguas residuales, minas de carbón, solidos sedimentables, metodología AHP.

\begin{abstract}
The filtration of surface and groundwater is very common when mining works is advancing, since underground aquifers, old blocks exploited must be crossed and within the extraction process, a settlement of the strata that produce some range of subsidence and fracture of these is generated, resulting in the filtration of these waters from the surface of the ground to inside of the mines. This wastewater must be evacuated back to the surface, which it is necessary to conduct it throughout the mining works (using mechanisms such as ditches, hoses and pipes), tending to contaminate with the environment of mining pollution and the same direct contact with the rocks of the excavations. Once driven to the surface, by means of pumps of great capacity and flow depending on the depth of the work, these wastewaters must be managed in treatment systems in order to perform a cleaning in this case of the concentrations of sediment solids and be able to be discharged to nearby sources, to be reused in processes other than human consumption. The present study evaluated in a technical, economic and environmental way three water treatment alternatives, getting results from alternative 1 Coagulation-
\end{abstract}


Flocculation, an efficiency percentage of $99.12 \%$, a total installation cost of $\$ 17,363,978$ Colombian money, and a low environmental affectation, simulating with the SuperDecisions ${ }^{\circledR}$ software, that Alternative 1 Coagulation-Flocculation has the ranking number 1 , followed by the alternative Activated Carbon with ranking number 2 and, finally the maintenance of the system with ranking 3.

Key Words: wastewater, coal mines, sedimentable solids, AHP methodology.

\section{INTRODUCCIÓN}

Para el trabajo de investigación, se realiza un diagnóstico del sistema actual de tratamiento de aguas residuales construido dentro del proyecto minero, el cual es comúnmente utilizado en las minas de la región, desde el punto de vista de los parámetros fisicoquímicos de calidad de agua y del diseño hidráulico de este, con el fin de evaluar sus falencias con respecto a la eficiencia del sistema y su diseño hidráulico. En la Resolución No. 0631 del 17 de marzo del 2015, se establecen los parámetros y los valores límites máximos permisibles que deben cumplir los vertimientos puntuales a los cuerpos de aguas superficiales y a los sistemas de alcantarillado público.

A partir de la normatividad vigente, se evalúan los parámetros de calidad de agua, para lo cual se realizó un muestreo y análisis inicial de las aguas residuales mineras con un laboratorio de aguas certificado, los resultados fueron tomados como base de comparación con la Resolución 0631 de 2015, con lo cual se determinó que el único parámetro que no cumple con la norma, es del Sólidos Sedimentables (SED). Así también, se evalúa técnicamente el diseño hidráulico del sedimentador principal desde sus dimensiones calculadas y construidas, así como, del caudal actual de la mina que alimenta el sistema.

En este sentido, para la reducción del parámetro de SED, se formulan tres alternativas técnicas para mejorar su eficiencia, las cuales se denominan Alternativa 1, Coagulación-Floculación; Alternativa 2, mantenimiento y limpieza del sistema de tratamiento actual y; Alternativa 3 , construcción de un filtro de carbón activado. Para cada una de estas alternativas se evalúa la eficiencia técnica, un análisis de costos y la afectación ambiental. De tal manera que, la elección provea la mejor alternativa que deba ser implementada; para lo cual se utiliza la metodología de Proceso de Análisis Jerárquico (AHP) por sus siglas en inglés, la cual usa una matriz de valoración de intensidad de importancia, que realiza una comparación paritaria, asignado según una escala de valores de uno (1) a nueve (9) y el grado de importancia de acuerdo a los mejores y peores resultados obtenidos en el proceso de cálculo de la eficiencia técnica, análisis de costos y afectación ambiental de cada alternativa, que toma como mejor opción la correspondiente al ranking 1. En últimas, la evaluación de técnicas presentada, tiene como fin que ingenieros ambientales, personal técnico y empresarios mineros de la zona, tengan una base que puedan replicar en sus unidades productivas, como solución para la reducción de SED, cumpliendo con el objetivo de mejorar la calidad del vertimiento, la aprobación de los permisos por parte de la autoridad ambiental y la calidad ambiental de la región.

\section{CONTEXTO GEOGRÁFICO}

El proyecto minero sitio de estudio, está ubicado en el municipio de Guachetá, Vereda "El Rabanal", departamento de Cundinamarca, dentro del área del título minero 1967t, de la empresa Minera Columbia Coal Company S.A. Para acceder al lugar se toma la vía principal que conduce de la ciudad de Bogotá al municipio de Ubaté y de este al municipio de Guachetá, con una distancia total de 118 Kilómetros. En la Figura 1 se observa la ubicación del área de estudio. El área de concesión minera tiene una extensión de 61 hectáreas, y se encuentra a una altitud entre los 2.620 a 2.900 metros sobre el nivel del mar con una temperatura media entre $12^{\circ}$ y $14^{\circ} \mathrm{C}$. Las aguas del vertimiento llegan a un canal efluente del río Lenguazaque, las cuales son utilizadas 
para diferentes procesos agroindustriales; por ejemplo, el regadío de pastos para semovientes, afectando no solamente el agua sino también al suelo y a los animales [1].

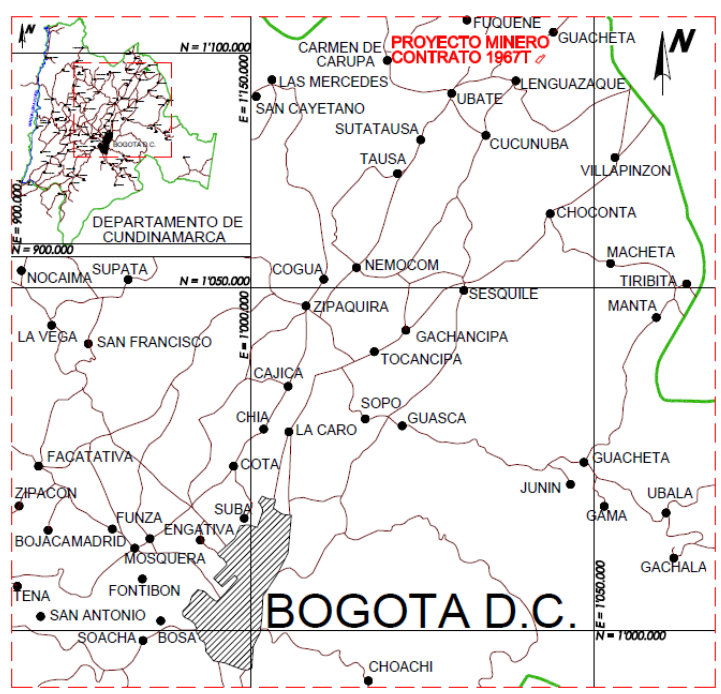

Figura 1. Ubicación del área del proyecto.

\section{MATERIALES Y MÉTODOS}

La elección de la mejor alternativa para la reducción de SED, inicia con un diagnóstico del sistema de tratamiento actual del proyecto, midiendo parámetros fisicoquímicos de calidad de agua, según la normatividad aplicada sobre aguas residuales en la industria minera y del diseño hidráulico del sistema en cuanto al cumplimiento de sus dimensiones. En la Figura 2, se observa la distribución del sistema de tratamiento del proyecto minero.

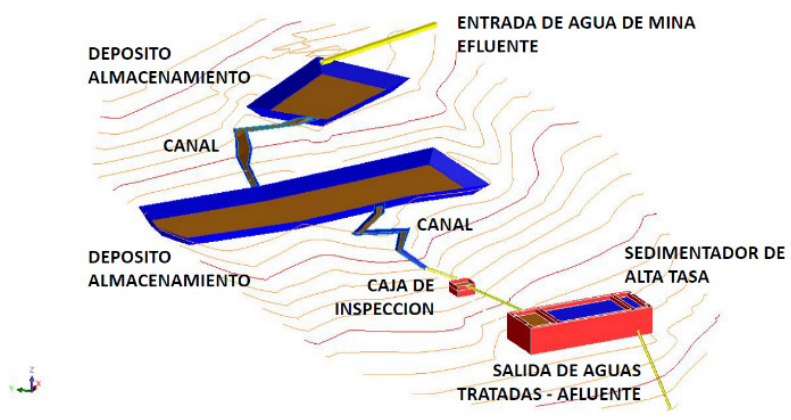

Figura 2. Sistema de tratamiento actual de aguas residuales.
Se evalúa técnicamente el diseño hidráulico del sedimentador principal, a partir de sus dimensiones y del caudal actual de la mina que alimenta el sistema. Para esta evaluación se utilizaron las fórmulas resumidas en la Tabla 1, según los parámetros que deben ser evaluados en el diseño.

Tabla 1. Fórmulas de diseño hidráulico cálculo tanque de sedimentación.

\begin{tabular}{|c|c|c|c|}
\hline $\begin{array}{l}\text { Nombre de } \\
\text { la Fórmula }\end{array}$ & Ecuación & Parámetros & $\mathbf{N}^{\circ}$ \\
\hline $\begin{array}{l}\text { Velocidad } \\
\text { de } \\
\text { Sedimen- } \\
\text { tación }\end{array}$ & $V_{s}=\frac{1}{18} g\left(\frac{\rho_{s}-1}{\eta}\right) d^{2}$ & $\begin{array}{l}\mathrm{V}_{\mathrm{s}} \text { : Velocidad de } \\
\text { sedimentación } \\
\text { (cm/seg) } \\
\mathrm{d} \text { : Diámetro de la } \\
\text { partícula }(\mathrm{cm}) \\
\eta \text { : Viscosidad cine } \\
\text { mática del agua } \\
\text { ( } \mathrm{cm}^{2} / \mathrm{seg} \text { ) } \\
\rho_{s} \text { : Densidad } \\
\text { relativa de carbón }\end{array}$ & (1) \\
\hline $\begin{array}{c}\text { Área } \\
\text { Superficial }\end{array}$ & $A_{s}=\frac{Q}{V_{\text {sed }}}$ & $\begin{array}{l}\mathrm{A}_{\mathrm{s}} \text { : Área superficial } \\
\left(\mathrm{m}^{2}\right) \\
\text { Q: Caudal de } \\
\text { diseño }\left(\mathrm{m}^{3} / \mathrm{seg}\right) \\
\mathrm{V}_{\text {sed }} \text { : Volumen del } \\
\text { sedimentador }(\mathrm{m})\end{array}$ & (2) \\
\hline $\begin{array}{l}\text { Velocidad } \\
\text { horizontal }\end{array}$ & $V_{H}=\frac{100 \times Q}{B \times H}$ & $\begin{array}{l}\text { Q: Caudal de } \\
\text { diseño }\left(\mathrm{m}^{3} / \mathrm{seg}\right) \\
\text { B: Ancho del } \\
\text { sedimentador }(\mathrm{m}) \\
\text { H: Altura del } \\
\text { sedimentador }(\mathrm{m})\end{array}$ & (3) \\
\hline $\begin{array}{l}\text { Tiempo de } \\
\text { retención }\end{array}$ & $\mathrm{T}_{0}=\frac{\mathrm{A}_{S} \times \mathrm{H}}{3600 \times \mathrm{Q}}$ & $\begin{array}{l}\mathrm{A}_{\mathrm{s}} \text { : Área superficial } \\
\left(\mathrm{m}^{2}\right) \\
\text { Q: Caudal de } \\
\text { diseño }\left(\mathrm{m}^{3} / \mathrm{seg}\right) \\
\mathrm{H} \text { : Altura del } \\
\text { sedimentador }(\mathrm{m})\end{array}$ & (4) \\
\hline
\end{tabular}




\begin{tabular}{|c|c|c|c|}
\hline $\begin{array}{l}\text { Número } \\
\text { de } \\
\text { orificios }\end{array}$ & $\begin{array}{l}\mathrm{A}_{0}=\frac{\mathrm{Q}}{\mathrm{V}_{0}} \\
n=\frac{\mathrm{A}_{0}}{\mathrm{a}_{0}}\end{array}$ & $\begin{array}{l}A_{o}: \text { Área total de } \\
\text { orificios }\left(\mathrm{m}^{2}\right) \\
Q: \text { Caudal de } \\
\text { diseño (m3/seg) } \\
V_{0} \text { : Velocidad de } \\
\text { orificios (m/seg) } \\
a_{0}: \text { Área de cada } \\
\text { orificio }\left(\mathrm{m}^{2}\right) \\
n: \text { número de } \\
\text { orificios }\end{array}$ & (5) \\
\hline $\begin{array}{l}\text { Área del } \\
\text { sedimen- } \\
\text { tador }\end{array}$ & $\mathrm{A}=\frac{V_{\text {Sed }}}{\mathrm{H}}$ & $\begin{array}{l}\mathrm{V}_{\text {sed }}: \text { Volumen del } \\
\text { sedimentador }\left(\mathrm{m}^{3}\right) \\
\mathrm{H}: \text { Altura del } \\
\text { Sedimentador }(\mathrm{m}) \\
\mathrm{A}: \text { Área del } \\
\text { sedimentador }\left(\mathrm{m}^{2}\right)\end{array}$ & (6) \\
\hline $\begin{array}{l}\text { Ancho del } \\
\text { sedimen- } \\
\text { tador }\end{array}$ & $\mathrm{a}=\sqrt{\frac{\mathrm{A}}{2}}$ & $\begin{array}{l}\text { a: Ancho de } \\
\text { sedimentador } \\
\text { A: Área del } \\
\text { sedimentador }\end{array}$ & (7) \\
\hline $\begin{array}{l}\text { Longitud } \\
\text { del } \\
\text { sedimen- } \\
\text { tador }\end{array}$ & $L=\frac{A}{a}$ & $\begin{array}{l}\text { L: Longitud del } \\
\text { sedimentador } \\
\text { A: Área del } \\
\text { sedimentador } \\
\text { a: Ancho de } \\
\text { sedimentador }\end{array}$ & (8) \\
\hline
\end{tabular}

\subsection{Alternativas de diseño para optimización técnica}

Se estudian tres alternativas para el mejoramiento de la calidad del agua, las cuales se denominan: coagulaciónfloculación, mantenimiento y limpieza del sistema de tratamiento actual y la construcción de un filtro de carbón activado. En la Figura 3, se observa el diagrama de flujo del procedimiento que se lleva a cabo para la evaluación de la eficiencia de las alternativas.

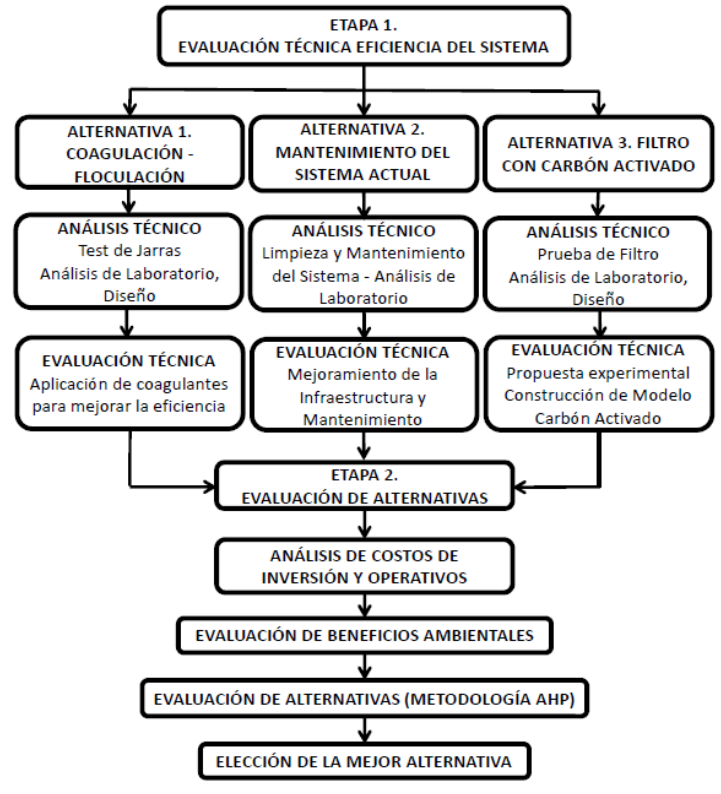

Figura 3. Diagrama de flujo para elección de alternativas.

Alternativa 1. Coagulación - Floculación. Se tomaron un total de diez (10) litros de agua los cuales se analizaron teniendo en cuenta las principales variables o parámetros de control de índole técnico de importancia dentro de la investigación, utilizando los respectivos instrumentos: $\mathrm{pH}$ (pH-metro), turbidez (Turbidímetro), sólidos sedimentables (cono Imhoff), y alcalinidad (titulación), en el laboratorio de aguas.

En el procedimiento de coagulación - floculación se realiza una prueba de test de jarras con el fin de observar el comportamiento de los sólidos frente a dos agentes coagulantes (sulfato de aluminio y cloruro férrico). Las variables a considerar en el diseño experimental son las características del Floc y su clasificación de acuerdo al índice Wilcomb, mediante la adición de Sulfato de Aluminio o Cloruro Férrico en cantidades de $1 \mathrm{~mL}, 3 \mathrm{~mL}$ y $5 \mathrm{~mL}$, comparando los resultados de los parámetros fisicoquímicos iniciales, y los resultados obtenidos después del ensayo de laboratorio. El procedimiento se lleva a cabo con los pasos que se observan en los diagramas de flujo de las Figuras 4 y 5 . 


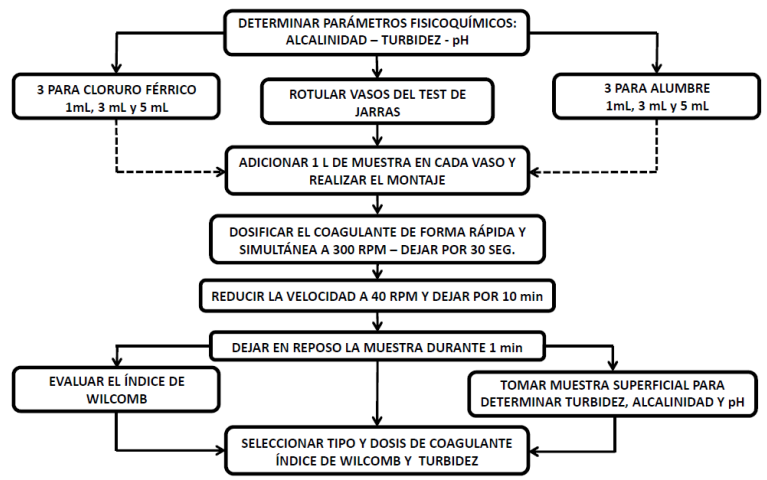

Figura 4. Diagrama de flujo prueba de jarras.

Se observa las características del Floc y se clasifica de acuerdo al índice de Wilcomb. (Ver Tabla 2 y Figura 6).

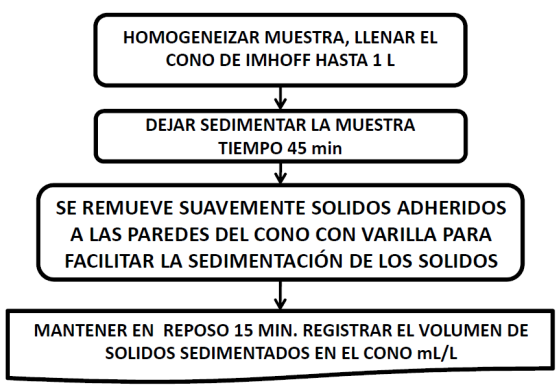

Figura 5. Diagrama de flujo prueba de cono Imhoff.

Tabla 2. Índice Wilcomb [2].

\begin{tabular}{cl}
\hline $\mathbf{N}^{\circ}$ & \multicolumn{1}{c}{ Descripción } \\
\hline 0 & Floc coloidal. Sin signo de aglutinación \\
\hline 2 & $\begin{array}{l}\text { Visible. Muy pequeño, casi imperceptible para } \\
\text { observadores no entrenados. }\end{array}$ \\
\hline & $\begin{array}{l}\text { Disperso. Bien formado, pero no uniformemente } \\
\text { distribuido. Sedimenta muy lentamente o no } \\
\text { sedimenta. }\end{array}$ \\
\hline 6 & $\begin{array}{l}\text { Claro. Floc de tamaño relativamente grande pero } \\
\text { que precipita con lentitud. }\end{array}$ \\
\hline 8 & $\begin{array}{l}\text { Bueno. Floc que se deposita fácil, pero no } \\
\text { completamente. }\end{array}$ \\
\hline 10 & $\begin{array}{l}\text { Excelente. Floc que se deposita todo dejando el } \\
\text { agua cristalina. }\end{array}$ \\
\hline
\end{tabular}

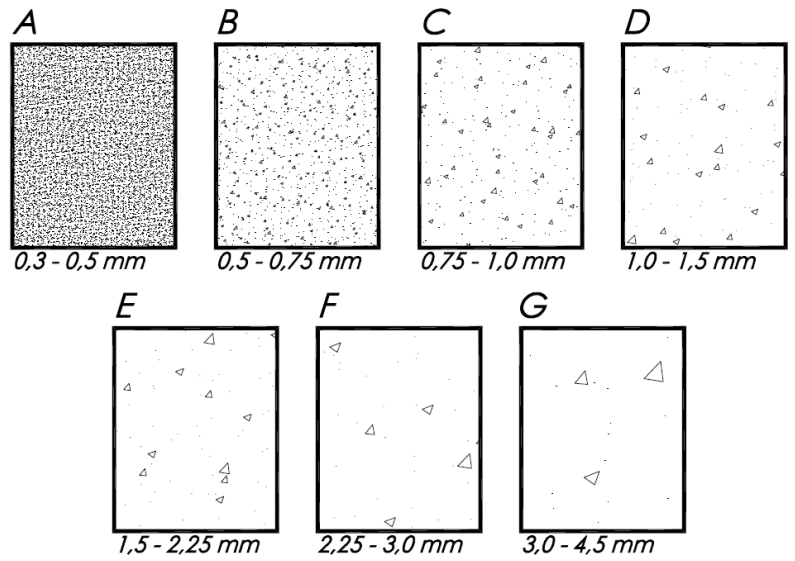

Figura 6. Índices de Wilcomb para determinar el tamaño del floculo [3].

Evaluación del tipo de coagulante: Se realiza el test de jarras y se evalúa el índice de Wilcomb, para los dos coagulantes adicionados, seleccionado el que mejor arrastre tenga para evaluar su dosis óptima.

Evaluación de la dosis óptima de coagulante: Una vez analizado y elegido del test de jarras, el coagulante cuyo resultado da el mayor valor de índice Wilcomb, se utiliza una dosis (en $\mathrm{mL}$ ) por encima y una por debajo, para calcular la dosis óptima de coagulante. Para la realización de los cálculos de los parámetros de Sólidos Sedimentables, dosis de solución de coagulante y titulación, se utiliza las ecuaciones descritas a continuación en la Tabla 3:

Tabla 3. Fórmulas para cálculo de parámetros sólidos sedimentables

\begin{tabular}{ccll}
\hline $\begin{array}{c}\text { Nombre de la } \\
\text { Fórmula }\end{array}$ & Ecuación & Parámetros & $\mathbf{N}^{\circ}$ \\
\hline $\begin{array}{c}\text { Sólidos } \\
\text { Sedimen- } \\
\text { tables } \\
\text { Totales }\end{array}$ & SST $=\begin{array}{l}W_{f}=\text { Peso Final } \\
\text { Vol. muestra } \\
\times 1000\end{array}$ & $\begin{array}{l}W_{i}=\text { Peso Inicial } \\
\text { de muestra }\end{array}$ \\
\hline
\end{tabular}




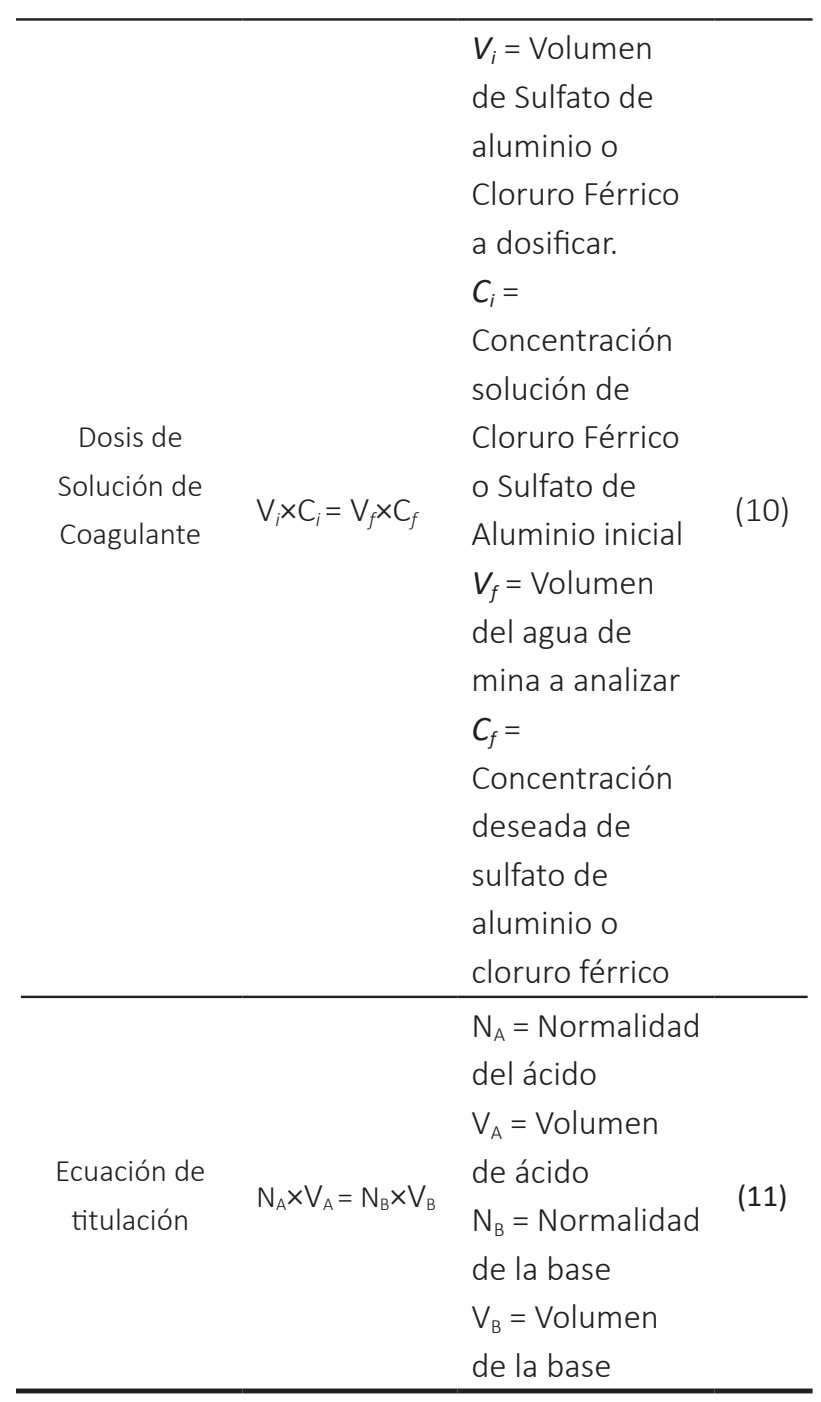

Cálculo de la eficiencia del sistema: Se calcula la eficiencia del sistema para la alternativa 1 , utilizando la ecuación (12) descrita a continuación, según los datos resultantes de turbiedad en el análisis de laboratorio y comparado con el dato de entrada antes de realizar los análisis de laboratorio.

$\underset{\text { Alternativa }}{\% \text { Eficiencia }}=1-\frac{\text { Turbiedad de Salida }}{\text { Turbiedad de Entrada }} \times 100 \%$

Alternativa 2. Mantenimiento del sistema actual. Se realiza el mantenimiento y limpieza del sistema actual de tratamiento de aguas residuales, retirando del sedimentador y los depósitos de almacenamiento los lodos existentes (ver Figura 7). Una vez se culmina la limpieza del sedimentador se realiza el llenado del mismo, y tiempo más tarde se vuelve a tomar una muestra puntual del agua considerando el sistema limpio, con fines de analizar su comportamiento. Se realiza el análisis de los parámetros de $\mathrm{pH}$, turbidez, sólidos sedimentables y alcalinidad, a la muestra tomada.

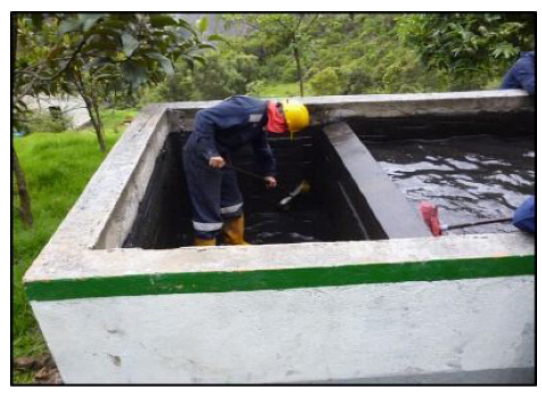

Figura 7. Mantenimiento y limpieza del sistema actual

Alternativa 3. Carbón Activado. Para la alternativa de carbón activado se construye y evalúa el montaje de un diseño experimental, el cual simula el paso del agua residual de la muestra tomada por un sistema de filtro de múltiples etapas, con el fin de obtener los parámetros de calidad del agua $y$, posteriormente, compararlos con los análisis de la caracterización inicial. Un Filtro Grueso Dinámico (FGDi) consiste en dos o más unidades compuestas de tres capas de grava de diferente tamaño, desde gruesa en la parte inferior hasta fina en la superficie.

El agua se filtra a través del lecho de grava desde la superficie hasta el fondo, alcanzando el sistema de drenaje, desde el cual fluye a la siguiente unidad de tratamiento. El diseño de un FGDi debe tener una velocidad de filtración entre 0,5 y 2,0 m/h de acuerdo a la Organización Panamericana de la Salud/Centro Panamericano de Ingeniería Sanitaria [4], debe estar ente 2 y $3 \mathrm{~m} / \mathrm{h}$. Se debe tener en cuenta las ecuaciones de la Tabla 4 y sus parámetros, para el diseño y construcción del filtro grueso dinámico como alternativa real para su tratamiento. 
Tabla 4. Fórmulas para cálculo del diseño de Filtro Grueso Dinámico FGDi.

\begin{tabular}{|c|c|c|c|}
\hline Nombre & Ecuación & Parámetros & $\mathbf{N}^{\circ}$ \\
\hline $\begin{array}{l}\text { Diámetro } \\
\text { del } \\
\text { tanque }\end{array}$ & $D=\sqrt{\frac{144 * Q}{\pi * V_{f}}}$ & $\begin{array}{l}\mathrm{Q}=\text { Caudal }(\mathrm{ml} / \mathrm{cm}) \\
\mathrm{Vf}=\text { Volumen de } \\
\text { filtración }(\mathrm{m} / \mathrm{h}) \\
144=\text { Factor de } \\
\text { corrección de } \\
\text { Unidades } \\
\mathrm{D}=\text { Diámetro }(\mathrm{cm})\end{array}$ & (13) \\
\hline $\begin{array}{l}\text { Velocidad } \\
\text { de } \\
\text { filtración }\end{array}$ & $V_{f}=\frac{144 * Q}{\pi * D^{2}}$ & $\begin{array}{l}\mathrm{Q}=\text { Caudal }(\mathrm{ml} / \mathrm{cm}) \\
\mathrm{Vf}=\text { Volumen de } \\
\text { filtración }(\mathrm{m} / \mathrm{h}) \\
144=\text { Factor de } \\
\text { corrección de } \\
\text { Unidades }\end{array}$ & (14) \\
\hline & & $\mathrm{D}=$ Diámetro $(\mathrm{cm})$ & \\
\hline
\end{tabular}

En la Figura 8, se observa el modelo a escala construido y utilizado en este diseño experimental, el filtro de carbón activado y su posterior evacuación del agua residual al almacenamiento para ser analizada de acuerdo con sus parámetros. En la construcción del diseño se realizó el tamizaje de los lechos filtrantes según la Norma Técnica Colombiana [5], pasándolos por mallas tamiz 8 para granulometría 2,73 mm (grava pequeña), y tamiz 1/2 pulgada para granulometría de 1,25 mm (grava gruesa). El carbón activado es de granulometría de 3 milímetros. A la muestra después del paso por el filtro, se le realiza el respectivo análisis de laboratorio de los parámetros de $\mathrm{pH}$, turbidez, sólidos sedimentables y alcalinidad, con el fin de calcular su eficiencia y poder comparar la alternativa con las otras dos alternativas propuestas.

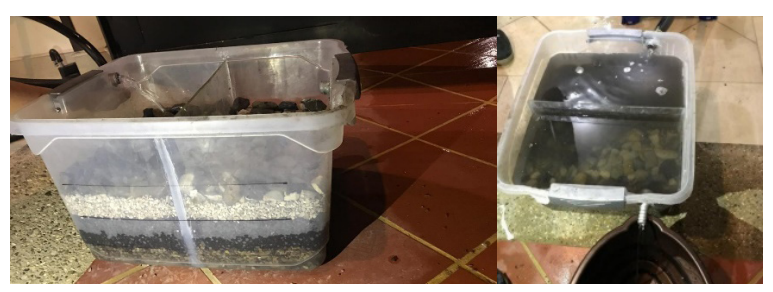

Figura 8. Esquema experimental tratamiento con carbón activado.

\subsection{Evaluación de Costos para las alternativas}

Se realizó la evaluación de los costos que interviene en cada una de las tres alternativas, tomando en cuenta las inversiones a realizar y los costos de operación, con el fin de ponderar cada alternativa desde el punto de vista de los costos totales para su evaluación y selección final. De esta manera, para los costos de inversión se tuvo en cuenta todos aquellos que implican la construcción de obras civiles, adquisición o alquiler de maquinaria y costos indirectos. Dentro de los costos de operación se toma en cuenta los costos en insumos, consumos de energía, mano de obra, impuestos, mantenimiento del sistema y construcción de obras.

\subsection{Evaluación Ambiental de Alternativas}

El método propuesto por Vicente Conesa FernándezVitoria [6], evalúa la importancia del impacto al valorar diferentes criterios de transformación (Ver Figura 9).

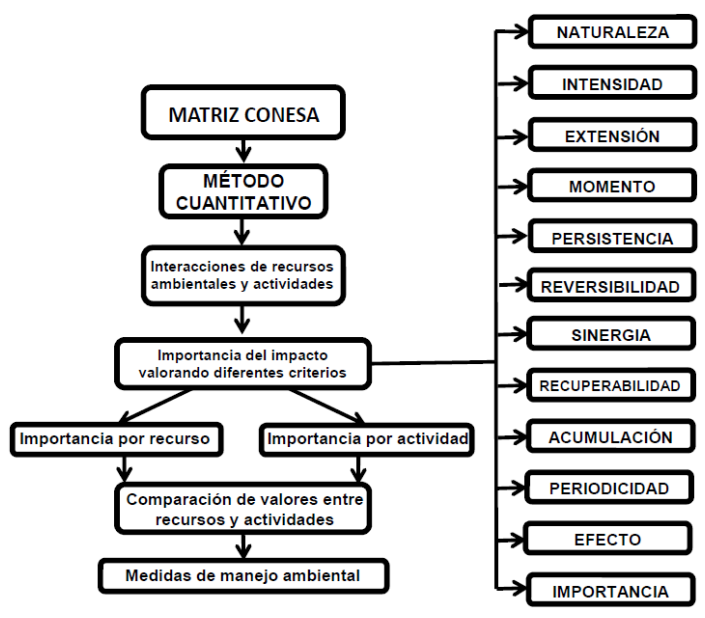

Figura 9. Diagrama del método de evaluación de impactos ambientales [6].

Las tres alternativas propuestas se evalúan con la matriz de Conesa, ya que este método permite valorar la importancia del impacto o afectación ambiental gracias a los criterios presentados en la Figura 9. Las siglas que representan los criterios de evaluación son: Naturaleza (N), Intensidad (IN), Extensión (EX), Momento $(\mathrm{MO})$, Persistencia (PE), Reversibilidad (RV), Sinergia $(\mathrm{SI})$, Acumulación (AC), Efecto (EF), Periodicidad (PR), Recuperabilidad (Mc), e Importancia (I). El valor de 
importancia o afectación ambiental se obtiene de acuerdo a los siguientes valores: Mínimo Valor: 17, Máximo Valor: 100, 17 - 40: Tipo C, 41 - 60: Tipo B y 61 - 100: Tipo A. En donde se le otorga mayor relevancia a la intensidad y a la extensión. La importancia se evalúa aplicando la ecuación 15.

$\mathrm{I}= \pm(3 I N+2 E X+M O+P E+R V+S I+A C+E F+P R+M C)$

De este modo, se evalúa cada una de las tres alternativas desde el punto de vista ambiental, utilizando como método una matriz que permite ver los impactos hacia factores ambientales de las posibles acciones a ejecutar en cada una de las alternativas, como resultado, se refleja la información en relaciones causa-efecto. Al final se obtiene la relevancia de cada alternativa y, de acuerdo a esta, se le asigna el tipo de afectación según el rango.

\subsection{Selección de la mejor alternativa}

De tal manera que, se pueda seleccionar la mejor alternativa, se utiliza la metodología de priorización de actividades denominada AHP (The Analytic Hierarchy Process), la cual fue desarrollada por Thomas L. Saaty [7], en los años 1971 a 1975 en Wharton School (Universidad de Pensilvania, Filadelfia, Estados Unidos), diseñado con el fin de resolver problemas complejos de criterios múltiples. Este método se basa en un sistema de comparación por pares donde se contrastan dos criterios bajo el esquema de medición. En la Tabla 5, se resume la valoración o intensidad de importancia que se le da en la comparación de criterios y alternativas.

Tabla 5. Importancia de escala.

\begin{tabular}{cl}
\hline Importancia Paritaria & \multicolumn{1}{c}{ Definición } \\
\hline 1 & Igual importancia entre las dos \\
\hline 3 & $\begin{array}{l}\text { Moderada importancia de uno } \\
\text { sobre otro }\end{array}$ \\
\hline 5 & Esencial o fuerte importancia \\
\hline 7 & Muy fuerte importancia \\
\hline 9 & Extrema importancia \\
\hline $2,4,6,8$ & Valores intermedios \\
\hline
\end{tabular}

El esquema de asociación de criterios y alternativas que utiliza la metodología AHP se muestra en la Figura 10.

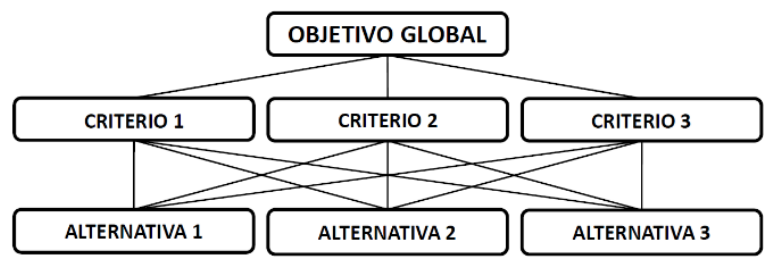

Figura 10. Esquema de comparación entre criterios y alternativas.

En este sentido, los criterios establecidos dentro del estudio, son determinados de acuerdo a las tres alternativas propuestas de tipo técnico (eficiencia del sistema), costos totales y afectación ambiental, los cuales permiten cumplir con el objetivo, el cual persigue la optimización de la Planta de Tratamiento de Aguas Residuales PTAR de la mina "Cuartas". De esta manera, los criterios aquí expuestos tienen aspectos cuantitativos y cualitativos, elementos que validan la toma de decisiones, así como, la jerarquización de alternativas. En la Tabla 6, se reúnen los criterios establecidos para el estudio, de acuerdo con lo señalado anteriormente.

Tabla 6. Criterios utilizados para la priorización.

\begin{tabular}{ccc}
\hline \multirow{2}{*}{ Criterio Evaluado } & \multicolumn{2}{c}{ Tipo de Asociación } \\
& Cualitativo & Cuantitativo \\
\hline $\begin{array}{c}\text { Eficiencia del } \\
\text { Sistema }\end{array}$ & $\mathrm{x}$ \\
\hline $\begin{array}{c}\text { Afectación } \\
\text { Ambiental }\end{array}$ & $\mathrm{x}$ & \\
\hline Costos Totales & & $\mathrm{x}$ \\
\hline
\end{tabular}

En concordancia, y para determinar la eficiencia del sistema, el valor tomado corresponde al porcentaje calculado de la eficiencia de remoción de turbidez para cada una de las alternativas. En la afectación ambiental, el valor tomado corresponde a la posible afectación negativa ejercida sobre el medio ambiente al ejecutar la actividad, esta es del tipo A Alta, tipo B Medio y tipo C Bajo. En el costo total el valor tomado corresponde al 
costo de la inversión, operación y mantenimiento que es requerida para llevar a cabo la puesta en marcha de cada actividad. La matriz construida se simula utilizando el Software SuperDecisions ${ }^{\circledR}$ V.3.2., y se presenta al final en los resultados, comparando las tres alternativas efectuadas, según el grado de importancia en concordancia con los resultados obtenidos en cada una de las alternativas. Enseguida, gracias a los valores obtenidos en la comparación de criterios, se hace la priorización de alternativas. Como consecuencia, la aplicación del método AHP para priorización de proyectos, al final brinda un resultado o ranking que permite establecer el orden de la prioridad de cada alternativa, de esta manera, es posible seleccionar la mejor alternativa a ejecutar.

\section{RESULTADOS}

En la Tabla 7, se presenta los resultados del análisis de aguas inicial, comparado con la Resolución 0631 del 17 de marzo de 2015, [8].

Tabla 7. Comparación parámetros fisicoquímicos versus norma.

\begin{tabular}{|c|c|c|c|}
\hline Parámetro & Unidades & $\begin{array}{l}\text { Extracción } \\
\text { de carbón } \\
\text { de piedra }\end{array}$ & $\begin{array}{c}\text { Resultados } \\
\text { Análisis }\end{array}$ \\
\hline $\mathrm{pH}$ & $\begin{array}{c}\text { Unidades } \\
\text { de } \mathrm{pH}\end{array}$ & 6,00 a 9,00 & 8,10 \\
\hline $\begin{array}{c}\text { Sólidos } \\
\text { Suspendidos } \\
\text { Totales (SST) }\end{array}$ & $\mathrm{mg} / \mathrm{L}$ & 50,00 & 26,00 \\
\hline $\begin{array}{c}\text { Sólidos } \\
\text { Sedimentables } \\
\text { (SED) }\end{array}$ & $\mathrm{mL} / \mathrm{L}$ & 2,00 & 4,30 \\
\hline $\begin{array}{l}\text { Alcalinidad } \\
\text { Total }\end{array}$ & $\mathrm{mg} / \mathrm{L} \mathrm{CaCO}_{3}$ & $\begin{array}{c}\text { Análisis y } \\
\text { Reporte }\end{array}$ & 295,00 \\
\hline
\end{tabular}

Cuando se realiza el análisis de la comparación con respecto a la calidad de agua, se observa que el único parámetro que no cumple con la norma es el de Sólidos Sedimentables (SED), con un valor resultante de $4,3 \mathrm{~mL} / \mathrm{L}$ y en donde la norma exige un total de $2 \mathrm{~mL} / \mathrm{L}$, para su cumplimiento.

4.1. Comprobación del diseño hidráulico del sistema actual

En la Tabla 8, se reúnen las dimensiones calculadas para el sedimentador y se compara con las dimensiones del actual sistema, para evaluar su capacidad y correcto diseño.

Tabla 8. Dimensiones actuales y calculadas del sedimentador.

\begin{tabular}{cccc}
\hline Dimensiones & $\begin{array}{c}\text { Sedimen- } \\
\text { tador } \\
\text { Actual }\end{array}$ & $\begin{array}{c}\text { Sedimentador } \\
\text { Calculado }\end{array}$ & $\begin{array}{c}\text { Ecuación } \\
\text { utilizada }\end{array}$ \\
\hline Área $(\mathrm{m} 2)$ & 14,5 & 11,6 & $(2)$ \\
\hline Ancho $(\mathrm{m})$ & 2,4 & 2,4 & $(7)$ \\
\hline Longitud $(\mathrm{m})$ & 6,12 & 5,2 & $(8)$ \\
\hline Altura $(\mathrm{m})$ & 1,2 & 2 & $(2)$ \\
\hline
\end{tabular}

\subsection{Parámetros fisicoquímicos del vertimiento}

Se realiza un análisis en laboratorio de sólidos sedimentables, $\mathrm{pH}$, temperatura y turbiedad, teniendo en cuenta sus resultados, de tal manera que, más adelante, se puedan contrastar las eficiencias técnicas de las alternativas. El resultado de los parámetros iniciales se resume en la Tabla 9, en donde se observa que el parámetro de los sólidos sedimentables da un valor de 5 $\mathrm{mL} / \mathrm{L}$ incumpliendo con la norma.

Tabla 9. Resultados parámetros fisicoquímicos del vertimiento sistema actual.

\begin{tabular}{cc}
\hline Parámetro & Valor Obtenido \\
\hline $\mathrm{pH}$ & 8,36 \\
\hline Temperatura $\left({ }^{\circ} \mathrm{C}\right)$ & 18,9 \\
\hline Turbiedad $(\mathrm{NTU})$ & 65 \\
\hline Sólidos Sedimentados $(\mathrm{mL} / \mathrm{L})$ & 5 \\
\hline
\end{tabular}


4.3. Evaluación técnica de alternativas de eficiencia del tratamiento

Alternativa 1. Coagulación - Floculación. El coagulante que presenta un mejor arrastre es el Cloruro Férrico 4000 partes por millón (PPM) en volumen de solución de $5 \mathrm{~mL}$; es decir, una concentración de 4000 mg/L. Los resultados obtenidos en laboratorio del test de jarras se ilustran en la Tabla 10.

Tabla 10. Índice Wilcomb obtenido para los coagulantes adicionados.

\begin{tabular}{cccc}
\hline Coagulante & $\begin{array}{c}\text { Volumen } \\
\text { de la } \\
\text { Solución a } \\
\text { aplicar }(\mathrm{mL})\end{array}$ & $\begin{array}{c}\text { Dosis de } \\
\text { coagulante } \\
\text { a aplicar } \\
\text { (mg/L) }\end{array}$ & $\begin{array}{c}\text { Índice } \\
\text { Observado }\end{array}$ \\
\hline Alumbre & 1 & 4 & 2 \\
\cline { 2 - 4 } (4000 PPM) & 3 & 12 & 4 \\
\cline { 2 - 4 } Cloruro & 5 & 20 & 4 \\
\cline { 2 - 4 } Férrico & 1 & 4 & 6 \\
\cline { 2 - 4 } (4000 PPM) & 3 & 12 & 6 \\
\hline
\end{tabular}

Evaluación de la dosis óptima de coagulante. Una vez se analiza y se elige el coagulante Cloruro Férrico en volumen de solución a aplicar de $5 \mathrm{~mL}$, dosis de coagulante de 20 $\mathrm{mg} / \mathrm{L}$, se calcula la dosis óptima tomando un volumen o cantidad de un (1) $\mathrm{mL}$ por encima y un (1) $\mathrm{mL}$ por debajo, los valores se resumen en la Tabla 11.

Tabla 11. Dosis óptima de coagulante Cloruro Férrico.

\begin{tabular}{cccc}
\hline \multirow{2}{*}{ Coagulante } & $\begin{array}{c}\text { Volumen de } \\
\text { la Solución a } \\
\text { aplicar (mL) }\end{array}$ & $\begin{array}{c}\text { Dosis de } \\
\text { coagulante } \\
\text { a aplicar } \\
\text { (mg/L) }\end{array}$ & $\begin{array}{c}\text { Índice } \\
\text { Observado }\end{array}$ \\
\hline Cloruro & 5 & 20 & 8 \\
\cline { 2 - 4 } Férrico & 4 & 16 & 6 \\
\cline { 2 - 4 } (4000 PPM) & 6 & 24 & 4 \\
\hline
\end{tabular}

Según los resultados obtenidos, el coagulante eficiente es el Cloruro Férrico con una dosis óptima de $20 \mathrm{mg} / \mathrm{L}$ y un índice Wilcomb de 8.

Alternativa 2. Mantenimiento del sistema actual. Después del mantenimiento del sistema actual de tratamiento de aguas, se llena nuevamente para posteriormente realizar al agua un análisis de parámetros fisicoquímicos, generando los resultados resumidos en la Tabla 12.

Tabla 12. Resultados finales mantenimiento sistema actual.

\begin{tabular}{cc}
\hline Parámetro & Valor Obtenido \\
\hline $\mathrm{pH}$ & 7,71 \\
\hline Temperatura $\left({ }^{\circ} \mathrm{C}\right)$ & 20,6 \\
\hline Turbiedad $(\mathrm{NTU})$ & 26,91 \\
\hline Alcalinidad $(\mathrm{PPM})$ & 9,5 \\
\hline
\end{tabular}

Alternativa 3. Prueba de filtro de carbón activado. En la Tabla 13, se resumen los resultados de los parámetros fisicoquímicos obtenidos una vez se pasa la muestra de agua por el diseño experimental del filtro de carbón activado.

Tabla 13. Resultados muestra filtro carbón activado.

\begin{tabular}{cc}
\hline Parámetro & Valor Obtenido \\
\hline $\mathrm{pH}$ & 5,0 \\
\hline Temperatura $\left({ }^{\circ} \mathrm{C}\right)$ & 20,0 \\
\hline Turbiedad $(\mathrm{NTU})$ & 3 \\
\hline Alcalinidad $(\mathrm{PPM})$ & 3,2 \\
\hline
\end{tabular}

4.3.1. Resumen de resultados de las alternativas. En la Tabla 14, se exponen los resultados obtenidos en laboratorio de los parámetros fisicoquímicos de las alternativas propuestas. 
Tabla 14. Resumen de resultados pruebas de laboratorio para las alternativas.

\begin{tabular}{cccccc}
\hline Alternativa & $\begin{array}{c}\text { PH } \\
\text { (Unidades } \\
\text { de PH) }\end{array}$ & $\begin{array}{c}\text { Tempe- } \\
\text { ratura } \\
\text { ('C) }\end{array}$ & $\begin{array}{c}\text { Turbie- } \\
\text { dad } \\
\text { (UTN) }\end{array}$ & $\begin{array}{c}\text { Alcalinidad } \\
(\mathbf{m g} / \mathbf{L} \text { de } \\
\mathbf{C a C O})\end{array}$ & $\begin{array}{c}\text { Eficiencia } \\
\text { del } \\
\text { Sistema } \\
\text { (\%) }\end{array}$ \\
\hline $\begin{array}{c}\text { Parámetros } \\
\text { Estado } \\
\text { Inicial }\end{array}$ & 8,36 & 18,9 & 65 & 10,5 & - \\
\hline $\begin{array}{c}\text { Coagulación } \\
-\end{array}$ & 7,67 & 19,9 & 0,57 & 9,2 & 99,12 \\
Floculación & & & & & \\
\hline $\begin{array}{c}\text { Limpieza } \\
\text { Sistema }\end{array}$ & 7,71 & 20,6 & 26,91 & 9,5 & 58,60 \\
Actual & & & & & \\
\hline Filtro & & & & & \\
Carbón & 5,00 & 20,0 & 3 & 3,2 & 95,38 \\
Activado & & & & & \\
\hline
\end{tabular}

4.4. Evaluación de costos para las alternativas

En la Tabla 15, se resume los resultados de los costos totales que se calculó para cada uno de los sistemas de tratamiento evaluados.

Tabla 15. Resumen de costos totales para alternativas.

\begin{tabular}{|c|c|c|c|}
\hline Alternativas & $\begin{array}{c}\text { Costos de } \\
\text { Inversión (\$) }\end{array}$ & $\begin{array}{c}\text { Costos de } \\
\text { Operación (\$) }\end{array}$ & $\begin{array}{c}\text { Costos Totales } \\
\text { (\$) }\end{array}$ \\
\hline $\begin{array}{l}\text { Coagulación - } \\
\text { Floculación }\end{array}$ & $\$ 13^{\prime} 893.750$ & $\$ 3^{\prime} 470.228$ & $\$ 17^{\prime} 363.978$ \\
\hline $\begin{array}{l}\text { Mantenimiento } \\
\text { Sistema Actual }\end{array}$ & 0,00 & $\$ 3^{\prime} 413.256$ & $\$ 3^{\prime} 413.256$ \\
\hline $\begin{array}{l}\text { Filtro de Carbón } \\
\qquad \text { Activado }\end{array}$ & $\$ 4^{\prime} 540.500$ & $\$ 8^{\prime} 455.192$ & \$12'995.692 \\
\hline
\end{tabular}

4.5. Evaluación de la afectación ambiental de las alternativas

En la Tabla 16, se realiza la comparación de los resultados de la valoración de la afectación ambiental y el valor de importancia para cada uno de los sistemas de tratamiento evaluados.
Tabla 16. Resumen de valoración ambiental para las alternativas

\begin{tabular}{ccc}
\hline Alternativas & $\begin{array}{c}\text { Valoración } \\
\text { Ambiental }\end{array}$ & $\begin{array}{c}\text { Valor de } \\
\text { Importancia }\end{array}$ \\
\hline $\begin{array}{c}\text { Coagulación }- \\
\text { Floculación }\end{array}$ & -29 & BAJO \\
\hline Limpieza Sistema Actual & -32 & BAJO \\
\hline Filtro Carbón Activado & -30 & BAJO \\
\hline
\end{tabular}

4.6. Selección de la mejor alternativa

En la Tabla 17, se resumen los resultados finales obtenidos en los tres criterios analizados (eficiencia del sistema, costos totales y afectación ambiental), para las alternativas propuestas; valores de tipo cualitativos y cuantitativos, base para realizar la comparación paritaria según la metodología AHP.

Tabla 17. Valoración cualitativa y cuantitativa según cada alternativa

\begin{tabular}{cccc}
\hline Criterios & Técnico & Ambiental & Económico \\
\hline Alternativas & $\begin{array}{c}\text { Eficiencia } \\
\text { del } \\
\text { sistema }\end{array}$ & $\begin{array}{c}\text { Afectación } \\
\text { Ambiental }\end{array}$ & $\begin{array}{c}\text { Costos } \\
\text { Totales }\end{array}$ \\
\hline $\begin{array}{c}\text { Coagulación - } \\
\text { Floculación }\end{array}$ & $99,12 \%$ & BAJA & \$17'363.978 \\
\hline $\begin{array}{c}\text { Limpieza } \\
\text { Sistema } \\
\text { Actual }\end{array}$ & $58,60 \%$ & BAJA & \$3'413.256 \\
\hline $\begin{array}{c}\text { Filtro de } \\
\text { Carbón } \\
\text { Activado }\end{array}$ & $95,38 \%$ & BAJA & $\$ 12^{\prime} 995.692$ \\
\hline
\end{tabular}

Para hacer el estudio de priorización de actividades se realiza un árbol de jerarquías para replicarlo en el software SuperDecisions ${ }^{\circledR}$ V.3.2.0 (Ver Figura 11). 
Figura 11. Árbol de jerarquías replicado en el software SuperDecisions ${ }^{\circledR}$.

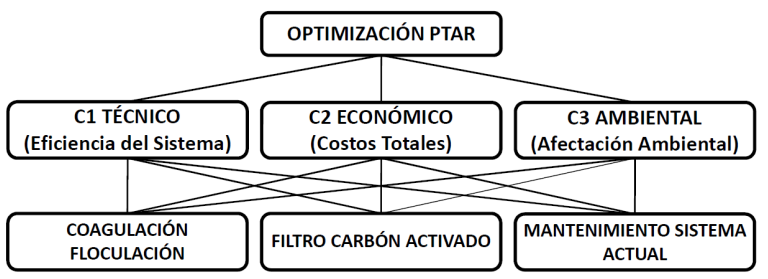

Los resultados de la comparación paritaria son presentados en la Tabla 18.

Tabla 18. Valores obtenidos de la comparación paritaria.

\begin{tabular}{ccccc}
\hline Criterio & $\begin{array}{c}\text { Afectación } \\
\text { Ambiental }\end{array}$ & $\begin{array}{c}\text { Costo } \\
\text { Total }\end{array}$ & $\begin{array}{c}\text { Eficiencia } \\
\text { del } \\
\text { Sistema }\end{array}$ & $\begin{array}{c}\text { Comparación } \\
\text { paritaria }\end{array}$ \\
\hline $\begin{array}{c}\text { Afectación } \\
\text { Ambiental }\end{array}$ & 1,0 & 7,0 & 9,0 & 0,048 \\
\hline Costo Total & 7,0 & 1,0 & 7,0 & 0,191 \\
\hline $\begin{array}{l}\text { Eficiencia } \\
\text { del Sistema }\end{array}$ & 9,0 & 7,0 & 1,0 & 0,761 \\
\hline
\end{tabular}

Los resultados de la priorización de alternativas se presentan en la Tabla 19 y Figura 12.

Tabla 19. Priorización de las alternativas.

\begin{tabular}{ccccc}
\hline Alternativa & $\begin{array}{c}\text { Eficiencia } \\
\text { del sistema }\end{array}$ & $\begin{array}{c}\text { Afectación } \\
\text { Ambiental }\end{array}$ & $\begin{array}{c}\text { Costos } \\
\text { totales }\end{array}$ & Ranking \\
\hline $\begin{array}{c}\text { Coagulación- } \\
\text { Floculación }\end{array}$ & 0,642 & 0,637 & 0,052 & 1 \\
\hline $\begin{array}{c}\text { Mantenimiento } \\
\text { del Sistema }\end{array}$ & 0,049 & 0,105 & 0,797 & 3 \\
\hline $\begin{array}{c}\text { Carbón } \\
\text { Activado }\end{array}$ & 0,309 & 0,258 & 0,151 & 2 \\
\hline
\end{tabular}

Figura 12. Resultados finales obtenidos del software SuperDecisions ${ }^{\circledR}$.

\begin{tabular}{||c||c|c|c|c|c|}
\hline Graphic & Alternatives & Total & Normal & Ideal & Ranking \\
\hline \hline & Coagulacion_Floculacion & 0.2644 & 0.5289 & 1.0000 & 1 \\
\hline \hline & Filtro_Carbon_Activado & 0.1381 & 0.2761 & 0.5221 & 2 \\
\hline \hline & Mantenimiento_Sistema_Actual & 0.0975 & 0.1950 & 0.3687 & 3 \\
\hline
\end{tabular}

Al exponer la muestra a los procesos de Coagulación Floculación (Alternativa 1) y Filtro de Carbón Activado (Alternativa 3), se precipitan los sólidos optimizando la eficiencia, la alcalinidad disminuye al igual que el pH, la temperatura aumenta de manera insignificante.

La alternativa más económica de acuerdo a los costos de inversión y operación es la alternativa 2, la alternativa 1 es la de mayor valor, debido a los costos de inversión en la construcción de las estructuras y en los insumos químicos utilizados. Por otro lado, la alternativa 3 es la que tiene el costo intermedio, no obstante, el costo con respecto a la alternativa 1 está casi a la par debido a las materias primas para la construcción del filtro de carbón activado.

A partir de la investigación, es posible afirmar que, todas las alternativas evaluadas ambientalmente tienen un valor de importancia tipo C (Bajo), en donde la alternativa 1 es la de mejor beneficio ambiental por tener el valor más bajo, seguida por la alternativa 3 y, la 2 consecutivamente en valores, según su grado de importancia.

El criterio de la eficiencia del sistema tiene una importancia del 0,761 o $76,1 \%$ por ciento, los costos totales tienen 0,191 o un $19,1 \%$ de importancia y la afectación ambiental con 0,048 o un 4,8\% por ciento de importancia, siendo el criterio de la eficiencia del sistema el factor determinante a la hora de tomar la decisión en la optimización del sistema de tratamiento de aguas residuales de la mina.

En la aplicación del método de decisión multicriterio AHP para la selección de la mejor alternativa para el estudio, da como resultado final un ranking que establece el orden de prioridad de las mismas. Lo cual da como resultado, según la simulación para este proyecto, que la Alternativa 1 Coagulación-Floculación tiene el ranking número 1, seguido de la alternativa Carbón Activado con ranking número $2 \mathrm{y}$, por último, el mantenimiento del sistema con ranking número 3 . Con base en lo anterior, se debe implementar al sistema actual de tratamiento de aguas residuales de mina, la Alternativa 1 CoagulaciónFloculación, de tal manera que, se optimice el proceso 
actual $y$, una vez puesto en funcionamiento, la calidad de agua tratada de vertimiento cumpla con las normas y reglamentos vigentes ambientalmente en Colombia.

\section{REFERENCIAS}

[1] Columbia Coal Company S.A., "Modificación del Programa de Trabajos y Obras (PTO) dentro del Contrato de Concesión No 1967T”, Guachetá, 2019.

[2] Metcalf \& Eddy, Inc., "Wastewater Engineering, Treatment and Reuse", Ed. New York: McGraw-Hill, 2003.

[3] J. Arboleda, "Manual de evaluación de impacto ambiental de proyectos, obras o actividades". Medellín, 2008.

[4] OPS/CEPIS. "Guía para diseño de sistemas de tratamiento de filtración en múltiples etapas", Organización Panamericana de la Salud, 2005. Disponible en: http://www. bvsde.opsoms.org/bvsacg/guialcalde/2sas/ d23/030_Construcci\%C3\%B3n_tratamiento_ Filtraci\%C3\%B3n_ME/Construcci\%C3\%B3n_ tratamiento_Filtraci\%C3\%B3n_ME.pdf

[5] ICONTEC. "Norma Técnica Colombiana 1522. Suelos ensayo para determinar la granulometría por tamizado", 1979.

[6] V. Conesa, "Guía metodológica para la evaluación del impacto ambiental", Ed. Mundi-Prensa, 2010.

[7] R.W. Saaty,"The Analytic hierarchy process - What it is and how it is used", Mathematical Modelling, pp. 161, 1987.

[8] Ministerio de Ambiente y Desarrollo Sostenible "Resolución No. 0631 del 17 de marzo del 2015", pp. 10-12, 2015. 